\title{
CT EVALUATION OF ASSOCIATION BETWEEN PERICARDIAL FAT VOLUME AND CORONARY ARTERY DISEASE
}

\author{
Sajay Alias ${ }^{1}$, Sholy K. Vareed ${ }^{2}$, Don Paul Mathew ${ }^{3}$, Justine Antony ${ }^{4}$ \\ ${ }^{1}$ Senior Resident, Department of Radiodiagnosis, SNIMS, Chalakka. \\ ${ }^{2}$ Associate Professor, Department of Radiodiagnosis, SNIMS, Chalakka. \\ ${ }^{3}$ Assistant Professor, Department of Radiodiagnosis, SNIMS, Chalakka. \\ ${ }^{4}$ Senior Resident, Department of Radiodiagnosis, SNIMS, Chalakka.
}

\begin{abstract}
BACKGROUND

Pericardial fat is the adipose tissue surrounding the heart. It releases inflammatory cytokines in a local fashion and affect only the adjacent structures, which are coronary arteries. Multiple studies have tried to establish the association of pericardial fat volume and calcium scoring with the incidence of coronary artery disease with contradicting results. We hope to provide additional evidence towards this already existing dispute and to comment whether pericardial fat volume measurement should be done as a routine diagnostic tool and consider it as a marker for ischaemic heart disease.

The objective of this study is to evaluate the association between pericardial fat volume and coronary artery disease using dual source computed tomography (DSCT), to evaluate the pericardial fat volume differences between patients with coronary artery disease and normal controls, to assess the relationship between pericardial fat volume and coronary calcium score and between different degrees of coronary artery stenosis.
\end{abstract}

\section{MATERIALS AND METHODS}

We included 117 DSCT coronary angiography studies, of which 62 cases were males and 55 were females; 74 scans showed positive findings of coronary artery disease and 43 cases were normal. Both the presence of coronary calcium score and/or coronary artery stenosis was taken into consideration as positive radiological findings of coronary artery disease. The scans were taken using Siemens Somatom definition flash DSCT with $100 \mathrm{kVp}, 0.75 \mathrm{~mm}$ slice thickness and mAs according to the patient's BMI. The scans were obtained in diastolic phase with the patient in supine position. Contrast agent used was Visipaque. Pericardial fat volume was measured in Siemens Somatom Syngo volume analysis software. The area of interest was taken as the areas of pericardial fat around the main coronary arteries and the threshold HU was kept between -30 and -190 HU. Coronary artery stenosis was assessed visually by two experienced radiologists and categorised to mild $(<50 \%)$, moderate $(50 \%-75 \%)$ and severe $(>75 \%)$. Coronary artery calcium score (Agatston score) was calculated using Siemens calcium score analysis software by an experienced radiologist. Each coronary artery was selected and the sum of all the calcium score of all the arteries were taken as total coronary calcium score. The data was written in excel format. All the statistics was done in SPSS 16.0 software.

\section{RESULTS}

The retrospective analysis of 117 CT coronary angiography scans revealed 74 (63.24\%) cases with positive findings for coronary artery disease either having coronary calcification or coronary artery stenosis. The mean of pericardial fat volume for normal cases was $76.84 \pm 35.79 \mathrm{~cm}^{3}$ and the positive cases with the evidence of coronary artery disease were $92.95 \pm 35.84 \mathrm{~cm}^{3}$. Significant differences were observed between the pericardial fat volume of normal subjects and subjects with the evidence of coronary artery disease in student $\mathrm{t}$-test $(\mathrm{P}=0.023)$. Pericardial fat volume was observed to have no significant difference between males and females $(p=0.191)$. Strong correlation was also revealed between age and pericardial fat volume $(r=0.275, p<0.05)$. Mean calcium score was $318.2 \pm 719.57$ in males and $104.92 \pm 261.78$ in females. Males were found to have more calcified plaques than females $(\mathrm{p}=0.04)$. The statistical studies revealed that there is a strict correlation between pericardial fat volume and coronary calcium score $(r=0.210, p=0.023)$. There was positive but insignificant correlation between pericardial fat volume and stenosis in total $(r=0.161, p=0.083)$; however, for different arteries showed significant associations between the pericardial fat volume and stenosis in right coronary artery $(r=0.264, p=0.004)$, left anterior descending artery $(r=0.177, p=0.056)$ and circumflex artery ( $r=0.105$, $p=0.262)$. Coronary artery stenosis was found to have a strong correlation with coronary calcium score $(r=0.452, p<0.001)$. A significant linear increase was seen in coronary calcium score with age $(p<0.05)$. Out of all the positive cases, left anterior descending artery was found to have the most calcified plaques as well as non-calcified soft plaques (89\%) causing stenosis. Right coronary artery (63.51\%) was the second to be affected, following it the circumflex arteries (40.54\%) and finally the left main coronary artery was involved.

\section{CONCLUSION}

Pericardial fat volume is strongly associated with coronary artery calcification and coronary artery stenosis, and thereby can be considered as a predictor or a risk factor for the development of coronary artery disease in the high-risk population. Further studies in this field are warranted, so that assessment of pericardial fat volume can be added as a routine diagnostic tool in cardiac CT.

\section{KEYWORDS}

Pericardial Fat, Coronary Artery Disease (CAD), Cardiac CT (Computed Tomography), CT Angiography, Agatston Calcium Score, Coronary Stenosis, Metabolic Syndrome, Adipose Tissue. 
HOW TO CITE THIS ARTICLE: Alias S, Vareed SK, Mathew DP, et al. CT evaluation of association between pericardial fat volume and coronary artery disease. J. Evolution Med. Dent. Sci. 2017;6(72):5117-5122, DOI: 10.14260/jemds/2017/1112

Financial or Other, Competing Interest: None.

Submission 20-07-2017, Peer Review 24-08-2017,

Acceptance 30-08-2017, Published 07-09-2017.

Corresponding Author:

Dr. Sajay Alias,

Senior Resident,

Department of Radiodiagnosis,

SNIMS, Chalakka, Ernakulam-683594,

Kerala

E-mail:drsajay@icloud.com

DOI: $10.14260 /$ jemds $/ 2017 / 1112$

\section{(c) (i) $(9)$}

\section{BACKGROUND}

Pericardial fat is the adipose tissue surrounding the heart, which consists of two layers: Epicardial (Fat compartment within the mediastinum) and paracardial (Fat compartment external to parietal pericardium) fat.[1] Association of pericardial fat volume with coronary artery disease is a disputed field with multiple studies supporting the claim and multiple of them which are against it. Obesity is a wellestablished risk factor for coronary artery disease. ${ }^{[2]}$ Distribution of body fat varies among individuals, gender and races. Many studies have proved that upper body distribution of adipose tissue has higher incidence of ischaemic heart diseases compared to the deposition of fat over the other regions.[3] Fat deposits in various parts of the body have different properties, which may signify the importance of fat distribution. For example pericardial fat, the fat depot around the heart was proved to release more inflammatory cytokines than subcutaneous adipose tissue. ${ }^{[4]}$ The inflammation caused by these inflammatory mediators is one of the causes of coronary artery disease.[5] These inflammations tend to occur only in local fashion and affect only the adjacent structures, ${ }^{[4]}$ which in this case are main coronary arteries. Therefore, pericardial fat volume is a principle interest while evaluating the risk factors for coronary artery disease.

Calcium scoring has been used as diagnostic routine in cardiac CT since few years after it being proved in multiple studies that it is clearly correlated with the presence of coronary artery stenosis. ${ }^{[6,7]}$ Coronary artery calcification (CAC) is an active part of the atherosclerotic process and predicts coronary artery disease (CAD) events in asymptomatic and symptomatic individuals.[6,7,8,9] Several cohort studies have been published, in which previously asymptomatic persons were studied with EBCT and followed up overtime for CAD events. These studies have reported that higher coronary calcium scores are associated with higher risks of CAD events.[10] The question of whether the calcium score adds incremental value to standard coronary artery disease risk factor assessment, however, remains controversial. Some researchers states that calcification may be merely a reflection of increased atherosclerosis conferred by standard CAD risk factors such as age, sex, cholesterol, blood pressure, smoking and diabetes. Calcification may actually reflect stabilisation and maturation of atherosclerotic plaques and hence lead to fewer myocardial infarctions and CAD deaths.[11-20]

In this study, we describe the association of pericardial fat volume with coronary artery disease showing its association between coronary artery stenosis and also coronary calcium score. Thereby, we hope to provide additional evidence towards the already existing dispute of whether or not the pericardial fat volume should be considered a risk factor or a predictor for coronary artery disease.

\section{MATERIALS AND METHODS \\ Study Design}

This is a descriptive study. A total number of 117 cardiac CT and coronary angiography were included in this study with retrospective evaluation. Among those, 74 scans were positive for the findings of coronary artery disease having either coronary calcification or coronary artery stenosis or both and 43 cases were normal. CT machines used was Siemens Somatom Definition Flash with $100 \mathrm{kVp}, 0.5 \mathrm{~mm}$ interslice gap and slice thickness of $0.75 \mathrm{~mm}$ with mAs varied by BMI. Non-contrast CT was used to evaluate calcium score and to measure pericardial fat volume while use of contrast was warranted to grade the coronary artery stenosis. Contrast agent used was Visipaque at a dose of $1 \mathrm{~mL} / \mathrm{kg}$ body weight, injected through the median cubital vein with a rate of $4.7-5 \mathrm{~mL} / \mathrm{s}$.

\section{Measuring Pericardial Fat Volume}

In the axial non-contrast cardiac CT, the section of origin of left main coronary artery was selected as a reference point (see Figure 1). Considering the interslice gap used, 30 slices superiorly and 60 slices inferiorly were selected as region of interest. Total length of this segment would be $4.5 \mathrm{~cm}$. This area was selected, because this area includes the adipose tissue around all the major coronary arteries. The software used for measuring pericardial fat volume was Siemens Syngo volume analysis. The threshold HU was kept between 30 and $-190 \mathrm{HU}$ for automatic delineation of fat density. The region of heart, which includes the pericardial fat was manually selected using a selection tool. Anterior limit for the selection was the chest wall and posterior limit was the descending aorta and the bronchus. Sum of all the 90 slices gives the total pericardial fat volume in $\mathrm{cm}^{3}$ (see Figure 2 - 4). The volume was measured twice and the average was taken to reduce the error as much as possible.

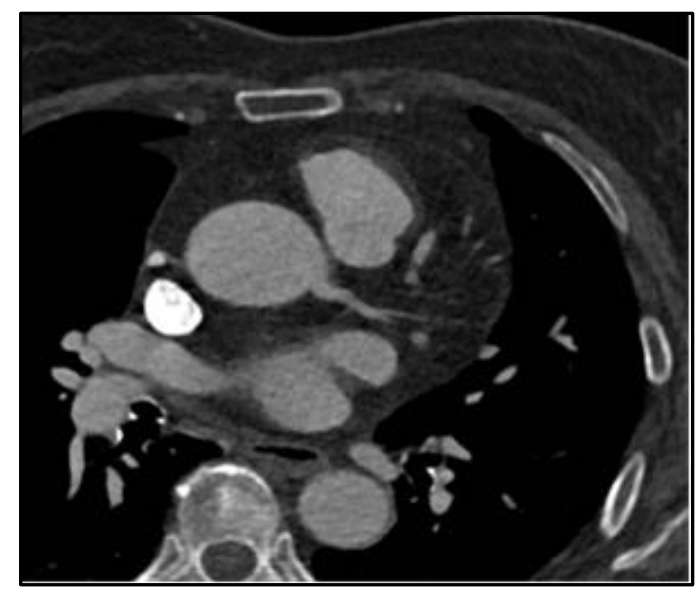

Figure 1. Axial CT showing the Origin of Left Main Coronary Artery, the Reference Point taken for the Measurement of Pericardial Fat Volume 


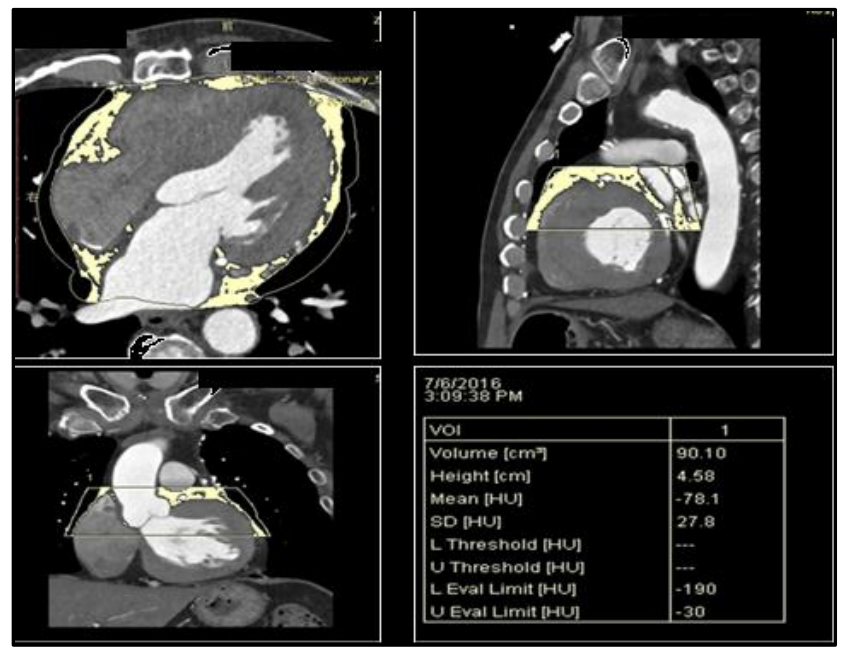

Figure 2. Measurement of Pericardial Fat Volume using Siemens Syngo Volume Analysis Software. The Result of the Volume Analysis is given on the Bottom Right Corner. Automatic Delineation of Fat is shown. Volume $=90.10 \mathrm{~cm}^{3}$

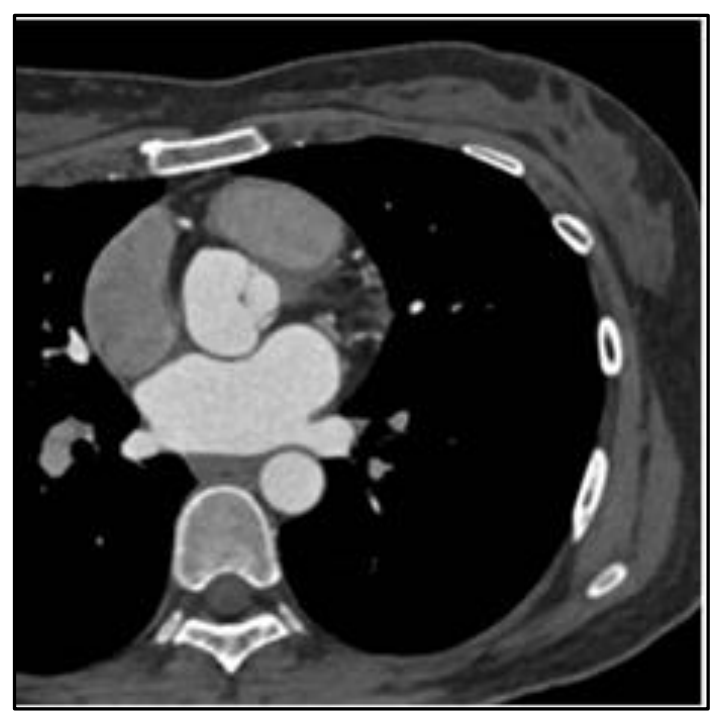

Figure 3. Axial CT Chest of 44-year-old Female Subject, showing Very Small Pericardial Fat Volume. The Volume was measured as $25.41 \mathrm{~cm} 3$

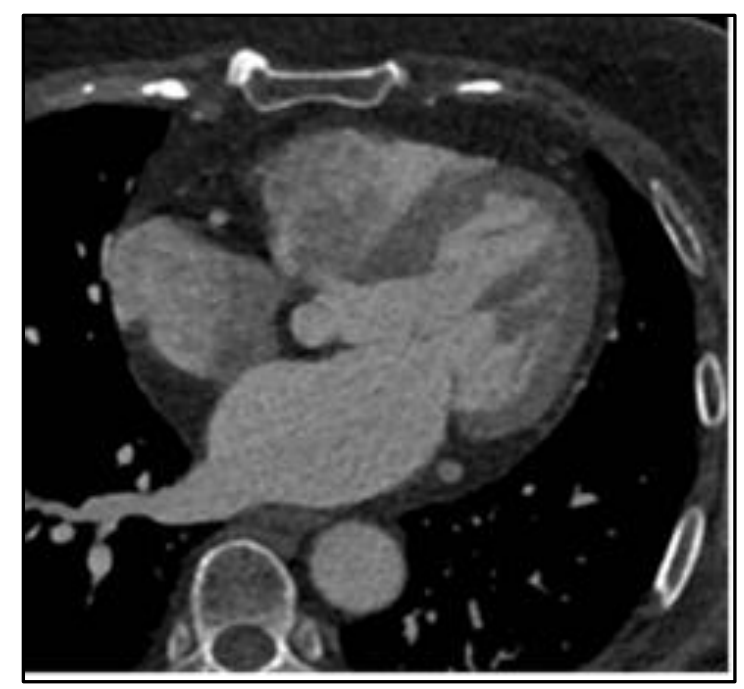

Figure 4. Axial CT Chest of 77-year-old Female Subject showing High Amount of Pericardial Fat Volume measuring $192.8 \mathrm{~cm} 3$
Measurement of Calcium Score

Non-contrast axial cardiac CT is used for calcium measurement. The software used is automatic calcium score measuring tool. Calcium score is measured by a single experienced technician in his routine workstation. Calcifications in individual arteries are selected and colour coded and the software adds the total calcium score after selection of all the calcified plaques in all the major coronary arteries (see Figure 5).

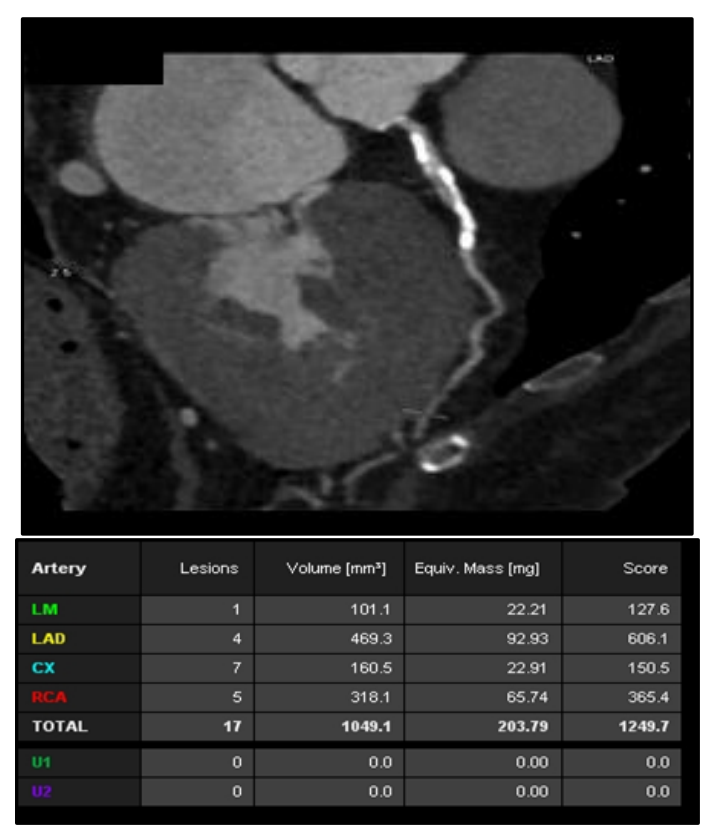

Figure 5. Shows Left Coronary Artery with Moderate Calcification. The Total Calcium Score is given in the Result at the Bottom. Calcium Score $=1249.7$

\section{Grading of Coronary Artery Stenosis}

Coronary angiography using Visipaque contrast agent at a dose of $1 \mathrm{~mL} / \mathrm{kg}$ body weight at a rate of 4.7 to $5 \mathrm{~mL} / \mathrm{sec}$ is done. The diastolic phase is selected since the coronary arteries are maximum dilated at this phase. Each main artery is reconstructed on its long axis. The stenosis is measured visually and cross checked by two experienced radiologists to grade them according to the degree of stenosis. Less than $50 \%$ narrowing is graded as mild stenosis, $50 \%$ to $75 \%$ is graded as moderate stenosis and above $75 \%$ narrowing is graded as severe stenosis (see Figure 6 - 8). Main coronary arteries selected are RCA, LAD artery and circumflex artery.

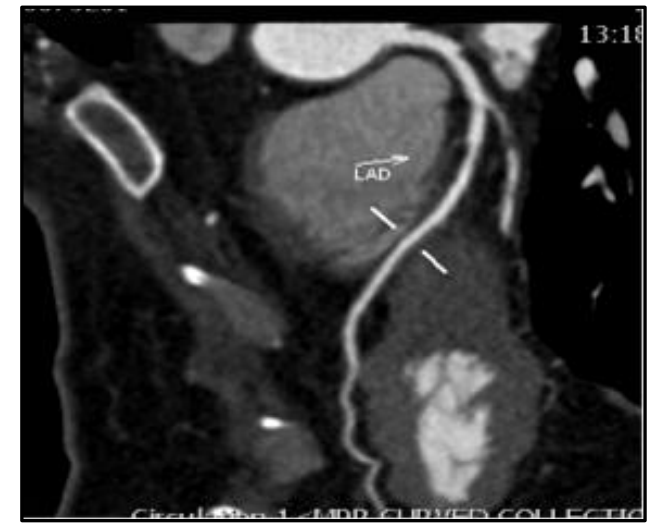

Figure 6. Reconstructed LAD Artery showing Mild Stenosis in the Proximal One-Third 


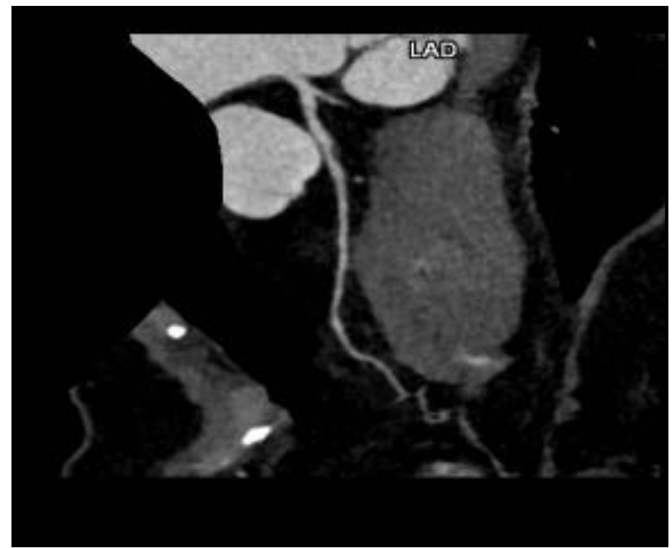

Figure 7. Reconstructed Left Anterior Descending Artery showing Moderate Stenosis in the Proximal Third

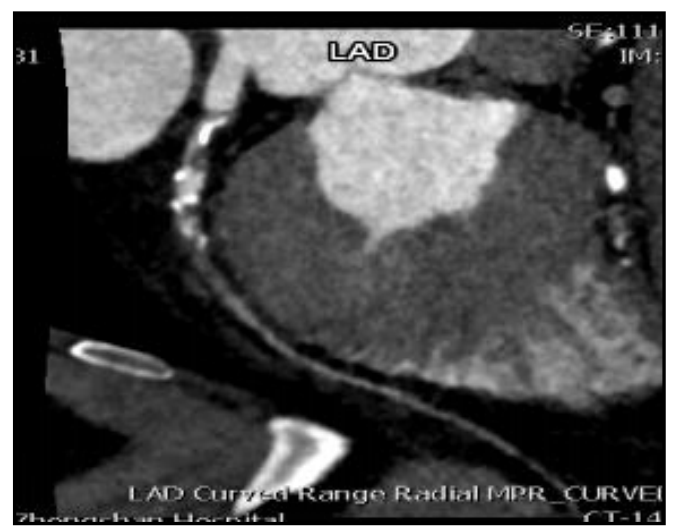

Figure 8. Reconstructed Left Anterior Descending Artery showing Severe Stenosis with Calcifications and Plaque

\section{Statistical Analysis}

All statistical analysis was done using SPSS version 16.0. Microsoft Excel was used to collect patient's data and the values obtained. Descriptive statistics were employed to describe the demographic variables. These include age, gender and the mean of the values obtained. The difference in pericardial fat volume with the presence of coronary artery disease was statistically tested using independent student $t$ test for non-normal distribution and one-way ANOVA test was used to test the differences of pericardial fat volume with different grades of stenosis in each coronary artery. Pearson product moment correlation coefficient test was used to show the correlation between pericardial fat volume and calcium score. Spearman's rank correlation coefficient was used to show the correlation between pericardial fat volume and the grade of stenosis in each artery. Bar chart and pie chart shown were statistically computer generated diagrams. A ' $p$ ' value of less than 0.05 was considered statistically significant.

\section{RESULTS}

\section{Pericardial Fat Volume of different Gender and Age}

74 out of 117 scans were positive $(63.24 \%)$ for the findings for coronary artery disease and $43(36.76 \%)$ were normal subjects. Out of 117 cases, 62 cases were males (83.78\%) and 55 cases were females (74.32\%). Mean age of males were $60.44 \pm 12.36$ and females were $64.2 \pm 10.09$. The youngest subject was a male of 27-year-old and oldest was 91 years. Strong correlation was observed between the age of the subjects and their pericardial fat volume $(r=0.275, p<0.05)$.
Mean pericardial fat volume for male subjects was $90.94 \pm$ $36.64 \mathrm{~cm}^{3}$ and mean pericardial fat volume in females was $81.73 \pm 38.99 \mathrm{~cm}^{3}$. The mean of pericardial fat volume for normal cases was $76.84 \pm 35.79 \mathrm{~cm}^{3}$ and those positive cases with the evidence of coronary artery disease were $92.95 \pm$ $35.84 \mathrm{~cm}^{3}$. Significant difference was observed between the pericardial fat volume of normal subjects and subjects with the evidence of coronary artery disease in student t-test $(\mathrm{P}=0.023)$. We observed pericardial fat volume increased significantly with age of the subjects and was also found to be more in male than female subjects.

Calcium Score and the Correlation with Pericardial Fat Volume

Out of the 74 positive cases, 50 cases had coronary calcification (67.57\%). Mean calcium score was $217.94 \pm$ 561.79. Males were found to have more calcified plaques than females $(p=0.04)$. Mean calcium score was $318.2 \pm 719.57$ in males and $104.92 \pm 261.78$ in females. A significant linear correlation was seen with age and the coronary calcium score ( $\mathrm{p}<0.05$ ). Numerically, highest calcium score found was 3877 in a scan of a 65 -year-old male patient. The statistical studies revealed that there is a strict correlation between pericardial fat volume and coronary calcium score $(r=0.210$, $\mathrm{P}=0.023$ ). Graph plotted using the values obtained showed a linear pattern (see Figure 10). Coronary calcium score was found to have a significant correlation with the presence of coronary artery stenosis $(\mathrm{r}=0.452, \mathrm{p}<0.001)$. LAD artery had the incidence of maximum calcified plaque in 49 cases with an average calcium score of $84.19 \pm 170.596$. RCA had the next incidence of calcification seen in 38 scans with a mean value of $65.19 \pm 242.878$. Following RCA circumflex artery showed the next incidence seen in 31 cases with a mean value of $6.83 \pm 15.535$ (see Figure 11).

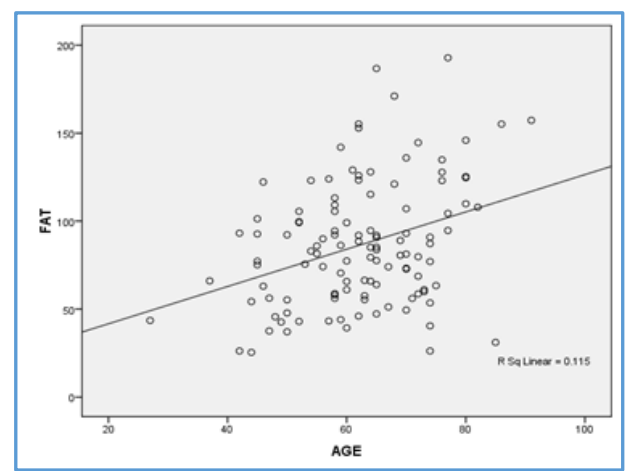

Figure 9. Line Graph showing the Significant Correlation between Pericardial Fat Volume and Age of the Subjects

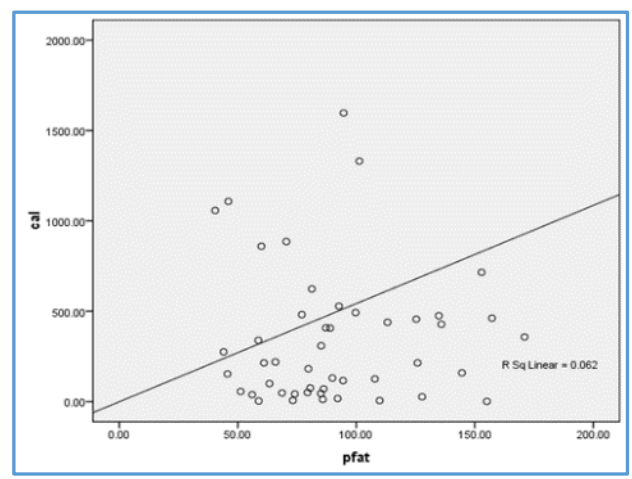

Figure 10. Line Graph showing Linear Correlation between Pericardial Fat Volume and Coronary Calcium Score 


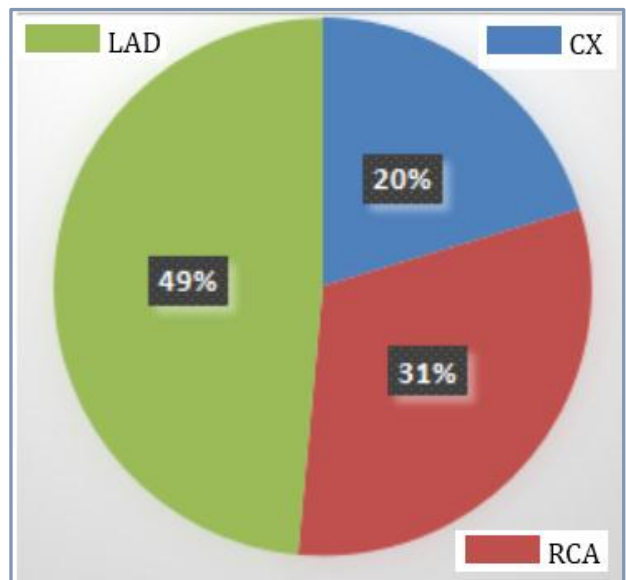

Figure 11. Pie Chart showing the Calcium Score of different Arteries

Coronary Artery Stenosis and the Correlation with Pericardial Fat Volume

70 cases were found to have some degree of stenosis in any of the coronary arteries; 48 out of 117 cases (66.66\%) were found to have both coronary artery stenosis and coronary calcification (see Figure 13 - 14). LAD artery was found to have most incidence of stenosis with 9 severe cases of stenosis, following which RCA was found to be second in the incidence of stenosis with 4 severe cases of stenosis. Circumflex arteries were found to have the least incidence of stenosis with just 2 cases of severe stenosis.

There was positive but insignificant correlation between pericardial fat volume and stenosis in total $(\mathrm{r}=$ $0.161, \mathrm{p}=0.083$ ); however, for different arteries statistical analysis found good correlation of pericardial fat volume with grades of coronary artery stenosis in RCA ( $r=0.264$, $p=$ 0.004). LAD ( $r=0.177, p=0.056$ ) (see Figure 12) and circumflex artery stenosis $(\mathrm{r}=0.105, \mathrm{p}=0.262)$ was correlated with pericardial fat volume, though not statistically significant enough. One-way ANOVA test performed showed significant difference in pericardial fat volume measured with the degree of stenosis in RCA and in circumflex artery $(p=0.009, p=0.002)$. However, stenosis in LAD arteries were not found to have significant difference with pericardial fat volume measured $(p=0.149)$. However, Post-hoc ANOVA test shows significant increase in pericardial fat volume in groups with mild/moderate/severe stenosis compared to groups with no stenosis in RCA and CX and LAD (See Table 1).

\begin{tabular}{|c|c|c|c|c|c|}
\hline & No Stenosis & Mild & Moderate & Severe & P-value \\
\hline \multirow{2}{*}{ RCA } & $78.80 \pm$ & $99.50 \pm$ & $83.52 \pm$ & $128.98 \pm$ & \multirow{2}{*}{0.009} \\
& 31.628 & 38.149 & $38.596^{*}$ & $51.259^{*}$ & \\
\hline \multirow{2}{*}{ LAD } & $81.67 \pm$ & $86.57 \pm$ & $94.46 \pm$ & $110.81 \pm$ & \multirow{2}{*}{0.149} \\
& 38.216 & 29.452 & 33.835 & $54.117^{*}$ & \\
\hline \multirow{2}{*}{ CX } & $85.71 \pm$ & $79.49 \pm$ & $124.69 \pm$ & $160.79 \pm$ & \multirow{2}{*}{0.002} \\
& 36.107 & 27.037 & $37.040 * @$ & $36.702^{*} @$ & \\
\hline
\end{tabular}

Table 1. Mean Pericardial Fat Volume in Different Arteries with their Grade of Coronary Artery Stenosis with their corresponding ANOVA Test Results

*The mean value is greater than the no stenosis group. @The mean value is greater than mild stenosis group.

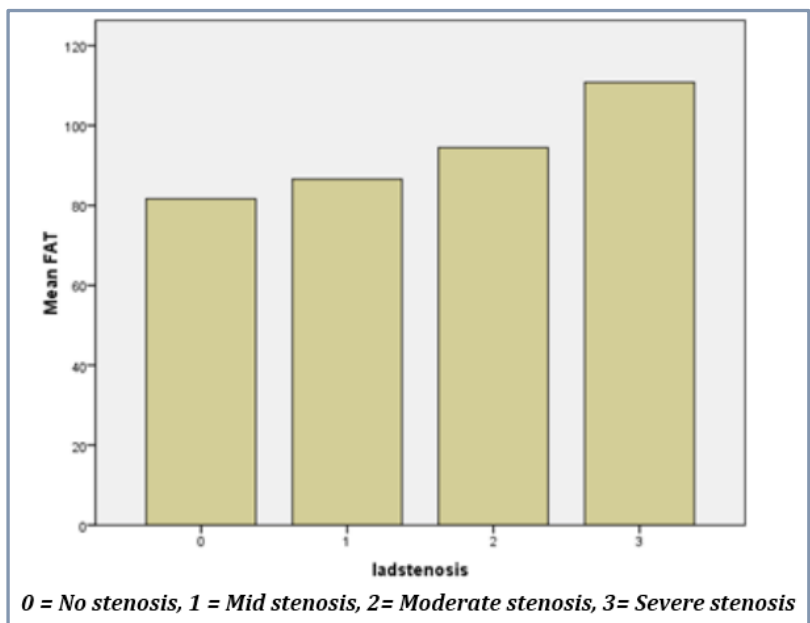

Figure 12. Bar Chart showing Increase in pericardial Fat volume as the Grade of Stenosis Increases
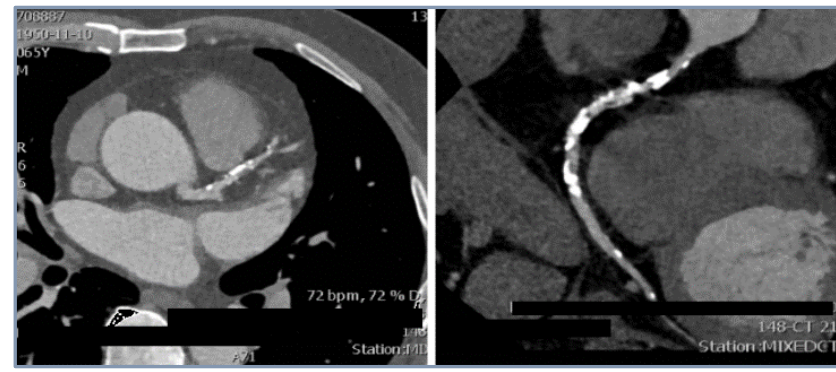

Figure 13. Contrast Enhanced Cardiac CT at the Level of Origin of Left Main Coronary Artery of a 65-year-old Male Patient with High Pericardial Fat Volume of $186.74 \mathrm{~cm} 3$ (Left) and Severe Stenosis in Reconstructed RCA (Right)

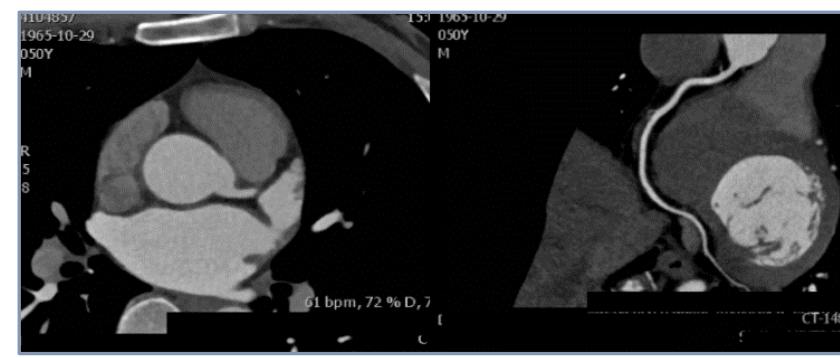

Figure 14. Contrast Enhanced Cardiac CT at the Level of Origin of Left Main Coronary Artery of a 50-year-old Male Patient with Low Pericardial Fat Volume of $37.1 \mathrm{~cm} 3$ (Left) and No Stenosis in the Reconstructed RCA

\section{DISCUSSION}

Our research on how efficient is the correlation of pericardial fat volume with coronary artery disease has fruitfully concluded that pericardial fat volume is related to the radiological evidence of coronary artery disease. We second the findings of many studies done in this field showing significant association between the adipose tissue around heart and the calcium score. We add up on that the association of pericardial fat volume with coronary artery stenosis as well, though not as strong as its association with calcified plaques. These results suggest that an increased pericardial fat volume may serve as a marker for severe atherosclerosis and might also be a risk factor for significant coronary artery disease in the general population. 
With the widespread use of multidetector CT scans, cardiac imaging is gaining more popularity recently on a population of which the commonest cause of death worldwide is cardiovascular diseases. Our study warrants the need of pericardial fat assessment to be added as a routine diagnostic tool for patients who undergoes cardiac CT. Measuring pericardial fat volume is an inexpensive and easy test to do. It does not require any more radiation dose than a cardiac CT would give, and can be measured by a routine axial cardiac CT taken while radiological workup for cardiovascular diseases, though it may be relatively time consuming.

\section{CONCLUSION}

Pericardial fat volume is strongly related to coronary artery disease. It shows even more strong association with coronary artery calcifications. LAD artery was found to be most commonly encountered with calcified as well as non-calcified soft plaque. Men had higher mean pericardial fat volume than women and pericardial fat volume was found to have a linear correlation with increasing age. Measuring pericardial fat must be considered as a routine diagnostic tool along with calcium scoring and coronary angiography, as it is an inexpensive and patient friendly procedure which consumes no extra radiational dosage and that it can be considered as an important predictor or a risk factor for coronary artery disease. Pericardial fat volume can be measured by ultrasound Echocardiography and cardiac MRI as well, if it is for the sake of screening so as to reduce radiational dose to the patient. Measuring pericardial fat volume can be very helpful in the diagnosis of ischaemic heart disease or to assess the high-risk population as well in screening for coronary artery disease.

\section{REFERENCES}

[1] Sacks HS, Fain JN. Human epicardial adipose tissue: a review. Am Heart J 2007;153(6):907-17.

[2] Eckel RH, Krauss RM. American heart association call to action: obesity as a major risk factor for coronary heart disease. AHA nutrition committee. Circulation 1998;97(21):2099-100.

[3] Rimm EB, Stampfer MJ, Giovannucci E, et al. Body size and fat distribution as predictors of coronary heart disease among middle-aged and older US men. Am J Epidemiol 1995;141(12):1117-27.

[4] Mazurek T, Zhang L, Zalewski A, et al. Human epicardial adipose tissue is a source of inflammatory mediators. Circulation 2003;108(20):2460-6.

[5] Hansson GK. Inflammation, atherosclerosis, and coronary artery disease. N Engl J Med 2005;352(16):1685-95.

[6] Detrano R, Hsiai T, Wang S, et al. Prognostic value of coronary calcification and angiographic stenoses in patients undergoing coronary angiography. J Am Coll Cardiol 1996;27(2):285-90.
[7] Arad Y, Spadaro LA, Goodman K, et al. Predictive value of electron beam computed tomography of the coronary arteries. 19-month follow-up of 1173 asymptomatic subjects. Circulation 1996;93(11):1951-3.

[8] Secci A, Wong N, Tang W, et al. Electron beam computed tomographic coronary calcium as a predictor of coronary events: comparison of two protocols. Circulation 1997;96(4):1122-9.

[9] Detrano RC, Wong ND, Doherty TM, et al. Coronary calcium does not accurately predict near-term future coronary events in high-risk adults. Circulation 1999;99 (20):2633-8.

[10] Blankenhorn DH. Coronary arterial calcification: a review. Am J Med Sci 1961;242:1-9.

[11] Lee TH, Brennan TA. Direct-to-consumer marketing of high-technology screening tests. $\mathrm{N}$ Engl J Med 2002;346 (7):529-31.

[12] Doherty TM, Wong ND, Shavelle RM, et al. Coronary heart disease deaths and infarctions in people with little or no coronary calcium. Lancet 1999; 353 (9146):41-2.

[13] Wong ND, Hsu JC, Detrano RC, et al. Coronary artery calcium evaluation by electron beam computed tomography and its relation to new cardiovascular events. Am J Cardiol 2000;86(5):495-8.

[14] Rumberger JA, Brundage BH, Rader DJ, et al. Electron beam computed tomographic coronary calcium scanning: a review and guidelines for use in asymptomatic persons. Mayo Clin Proc 1999;74 (3):243-52.

[15] Raggi P, Callister TQ, Cooil B, et al. Identification of patients at increased risk of first unheralded acute myocardial infarction by electron-beam computed tomography. Circulation 2000;101(8):850-5.

[16] Arad Y, Spadaro LA, Goodman K, et al. Prediction of coronary events with electron beam computed tomography. J Am Coll Cardiol 2000;36(4):1253-60.

[17] Yang T, Doherty TM, Wong ND, et al. Alcohol consumption, coronary calcium, and coronary heart disease events. Am J Cardiol 1999;84(7):802-6.

[18] Raggi P, Cooil B, Callister TQ. Use of electron beam tomography data to develop models for prediction of hard coronary events. Am Heart J2001;141(3):375-82.

[19] Vliegenthart R, Oudkerk M, Song B, et al. Coronary calcification detected by electron-beam computed tomography and myocardial infarction: the rotterdam coronary calcification study. Eur Heart J 2002;23(20):1596-603.

[20] Rumberger JA, Sheedy PF, Breen JF, et al. Coronary calcium, as determined by electron beam computed tomography, and coronary disease on arteriogram. Effect of patient's sex on diagnosis. Circulation 1995;91(5):1363-7. 